\title{
A New Concept of Power Control in Cellular Systems Reflecting Challenges of Today's Systems
}

\author{
Jonas Blom, Fredrik Gunnarsson and Fredrik Gustafsson \\ Department of Electrical Engineering \\ Linköpings universitet, SE-581 83 Linköping, Sweden \\ WWW: http://www.control.isy.liu.se \\ Email: jb@isy.liu.se, fred@isy.liu.se, \\ fredrik@isy.liu.se
}

April 16, 1999

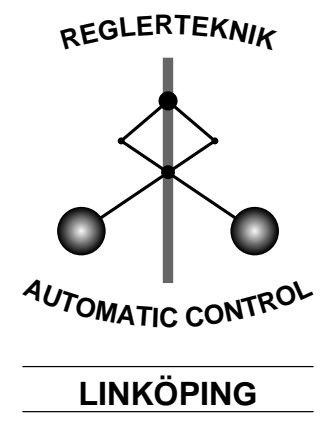

Report no.: LiTH-ISY-R-2143

Submitted to GLOBECOM'98, Sydney, Australia

Technical reports from the Automatic Control group in Linkping are available by anonymous ftp at the address ftp.control.isy.liu.se. This report is contained in the compressed postscript file 2143.ps.Z. 



\begin{abstract}
When the systems evolved from analog to digital, the performance was improved by the use of power control on the one hand and different modulations and coding schemes on the other. Condensing the available information we are able to propose a new concept of power control. The concept is applicable to real systems, since it uses the available measurements for estimating parameters necessary for the power control. It also supports the use of an adequate quality measure together with a quality specification supplied by the operator. We will use frequency hopping GSM as an example and the resulting control algorithm is ready for implementation in the software in the base stations where the output powers are computed. No modifications are needed in the GSM standard, the mobile terminals, the radio interfaces or in the base station transmitters. Finally we provide simulation results confirming the benefits of using the new concept for power control.
\end{abstract}

Keywords: Cellular radio systems; Power Regulators; Power Control Algorithms; ML Estimation; Quality mapping; Frame erasure rate 


\section{Introduction}

Several methods and strategies to control the power in cellular radio systems have been proposed $[18,17,5,6,1,15,7,16,3]$. Based on some simplifying assumptions, corresponding convergence results have been established. In most previous work rather ideal cases are considered. When considering power control in real systems, we find the following aspects interesting.

Quality Measure. Speech quality is a very subjective quantity. People have argued that $\mathrm{C} / \mathrm{I}$ is an adequate objective measure, and it has been used extensively in previous works, even though it is far from ideal.

Available Measurements. Usually the measurements are given in reports comprising a Quality Indicator (QI), reflecting the quality and a Received Signal Strength Indicator (RSSI), reflecting the received signal strength at the receiver. These values are coarsely quantized in order to use few bits.

Constraints. The output power levels are limited to a given set of values due to hardware constraints. This includes quantizing and the fact that the output power has an upper and a lower limit.

Time Delays. Measuring and control signaling take time, which results in time delays in the network.

In this paper the focus will be on how to handle quality specifications and the available measurements. We propose a concept for resolving these problems. For a more thorough discussion about the concept, we refer to $[4,8]$. The problems with constraints and time delays are the subject of the accompanying paper [9].

The surrounding environment as seen by the power controller, can be described as in Figure 1. Note that the chosen quality measure is not necessarily measurable directly.

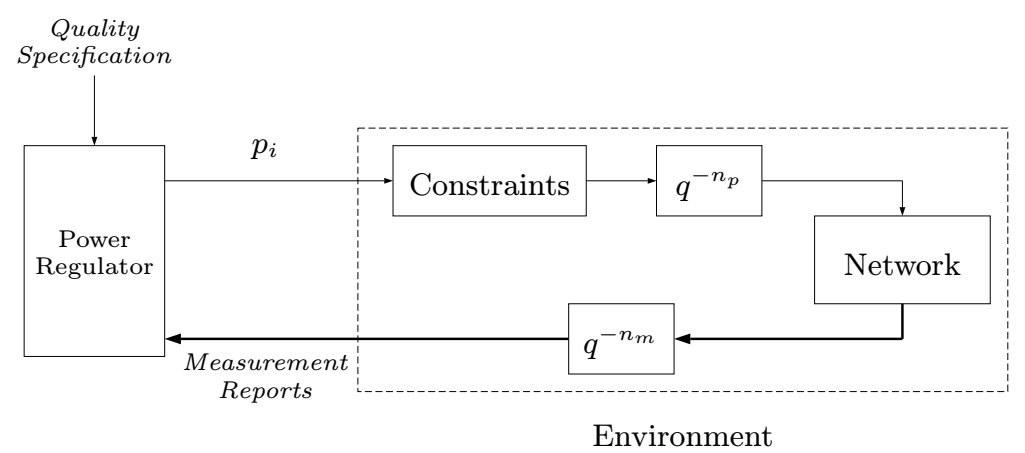

Figure 1: The surrounding Environment as seen by a decentralized controller, when considering time delays and constraints. The Network block incorporates the effects caused by the radio channel, such as power gain, noise and interfering transmitters. $p_{i}$ is the output power, while $n_{p}$ and $n_{m}$ represent delays.

When the algorithmic properties of the power controller, e.g. convergence and settling time, are to be studied, it may be easier to assume that the inter- 
esting values are at hand, and that quality is related to simple measures. Then we refer to this power controlling component as the Power Control Algorithm (PCA). This includes most of the algorithms developed in this area to date. On the other hand when discussing a complete solution that fits into the interfaces of existing systems, we use the term Power Regulator (PR).

\section{A New Concept of Power Control}

The proposed Power Regulator is comprising three parts as within the dashed lines in Figure 2. Altogether, given measurement reports, it will update the transmitter power in order to meet the specified quality. All transmitter powers in the network can be controlled by identical regulators. However it is important to note that even though the regulators are identical, they adapt to the individual situations experienced at each receiver, in order to achieve the specified quality.

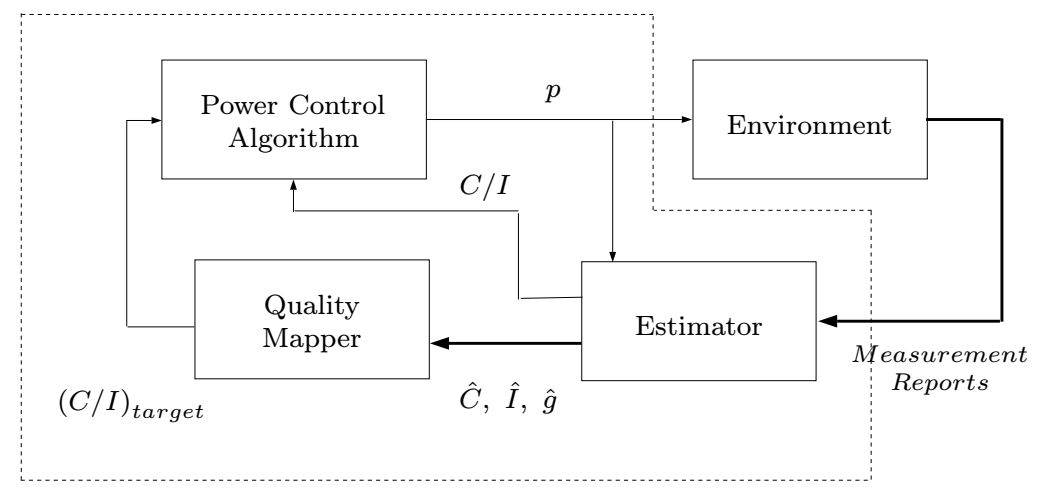

Power Regulator

Figure 2: The proposed Power Regulator can be divided into the three parts: Estimator, Quality Mapper and Power Control Algorithm. All transmitter powers in the network are controlled by identical Power Regulators, but each of them is adapting to the individual situation.

\subsection{The Estimator}

The problem of estimating the $\mathrm{C} / \mathrm{I}$ was addressed in $[2,14]$, but that approach assumes that analog signal strength measurements are available. In a real situation, the available information is given in measurement reports and one of the core problems is to extract as much relevant information as possible from these reports. Therefore we introduce an Estimator which will estimate the carrier $C$, the interference distribution $I$, and compute the path loss $g=C-p$ and the $C / I$. The information available to the estimator are the RSSI and the QI in the measurement reports, which in turn are depending on the parameters to be estimated. Hence, the measurement reports contain information from two 
conceptually different information sources. The estimator can be constructed in numerous ways, but we have chosen a Maximum Likelihood (ML) estimator, since it is successfully enables this data fusion and is an implementationally simple algorithm.

The method of ML estimation is based on a simple idea. Different probability density functions generate different data samples and any given data sample is more likely to have come from a particular distribution than from others. We will not go into the basic details of how ML estimation is implemented, instead, we refer the reader who is not familiar with these concepts to [10].

Let $x_{t}$ denote a measurement at time $t, \theta$ the parameters describing the interference distribution together with the carrier. The likelihood of a single measurement, $f_{X}\left(x_{t} ; \theta\right)=f_{1}\left(x_{t} ; \theta\right) \cdot f_{2}\left(x_{t} ; \theta\right)$ is a product of two likelihoods; one for the RSSI and one for the QI.

An adaptive algorithm (one that is able to track a varying parameter) should be used for ML estimation. Since the latest measurements contain more current information, the adaptive algorithm used should rely more on them. In order to accomplish this adaptive use of the latest measurements, a forgetting factor, $\lambda$, is introduced in the likelihood function $l_{t}(\theta)$

$$
l_{t}(\theta)=f_{X}\left(x_{t} ; \theta\right) f_{X}\left(x_{t-1} ; \theta\right)^{\lambda} \cdots f_{X}\left(x_{1} ; \theta\right)^{\lambda^{t-1}}
$$

Using $\lambda=0$ corresponds to "maximal forgetting", i.e. only the last measurement is considered, and $\lambda=1$ gives equal weight to all measurements. The following recursive expression can be used to implement the exponential "forgetting"

$$
\log l_{t}(\theta)=\log f_{X}\left(x_{t} ; \theta\right)+\lambda \log l_{t-1}(\theta)
$$

The estimate, $\theta$ is then obtained as the values which maximize the likelihood function. In order to obtain smooth estimates, it is preferable to filter each of the estimates separately, since they change at different rates. Exponential filtering is suitable for this purpose, see [9].

\subsection{The Quality Mapper}

There are a wide range of quality measure candidates, ranging from Bit Error Rate (BER) and Frame Erasure Rates (FER) to measures based on subjective listener tests. Regardless of which of these we choose, it can be desirable for the network operator to be able to specify the quality using a measure that reflects the tradeoff between capacity and speech quality. This is implemented in the Quality Mapper which acts as the glue between the estimator and the power control algorithm, by mapping the chosen quality measure onto a target $C / I$ using the estimated parameters.

When the power regulator is in use, the quality mapper calculates appropriate target $\mathrm{C} / \mathrm{I}$ values from the estimated quantities. From this point of view, the quality mapper is nothing but a look-up table. This table can be constructed from a quality function together with a specification of the required quality prior 
to the start-up of the system. Assume that the quality measure values can be described by a quality function

$$
Q\left(\gamma ; \theta_{1}, \ldots, \theta_{n}\right)
$$

where $\gamma$ denotes the $\mathrm{C} / \mathrm{I}$ and $\theta_{1}, \ldots, \theta_{n}$ denote quantities parameterizing the interference distribution. This function, which describes an objective quality measure, may be obtained from a model or from measurements.

If $Q_{0}$ denote the limit between acceptable and unacceptable quality, it is required

$$
Q\left(\gamma ; \theta_{1}, \ldots, \theta_{n}\right) \leq Q_{0}
$$

in order to get acceptable quality. It has been assumed (without loss of generality) that a lower value of $Q$ corresponds to better quality. If $Q$ is invertible and monotonically decreasing in $\gamma$, this can be solved simply by controlling $\gamma$ to fulfill

$$
\gamma \geq Q^{-1}\left(Q_{0} ; \hat{\theta}_{1}, \ldots, \hat{\theta}_{n}\right),
$$

The right-hand side implements the necessary quality mapping. In practice, the estimates are used in the quality mapper, as indicated in the equation above. The case where the quality function is non-invertible is solved in [4].

\subsection{The Power Control Algorithm}

When the solution is broken down into three parts, the functionality of the power control algorithm is to assign the appropriate power level so that the measured $\mathrm{C} / \mathrm{I}$ will track the target $\mathrm{C} / \mathrm{I}$. When an estimated $C / I$ and a target $C / I$ are available, several proposed algorithms are applicable, for instance the Distributed Power Control (DPC) algorithm [5], the algorithm proposed by Almgren, Andersson and Wallstedt (AAW) in [1] and a PID controller [4]. For a more thorough discussion about these algorithms, we refer to $[4,9]$.

\section{Example: Frequency Hopping GSM}

Note that the methods discussed this far are general, but in this section we will refine them, and apply them to frequency hopping GSM. As discussed in Section 2, the interference distribution must be parameterized by a number of parameters. In order to find these, we used a simulation model where it is assumed that the interference is constant during a burst. The gains of the transmitted powers in the random frequency hopping network were modeled by the path loss, shadow fading and Rayleigh fading. Thermal noise was also included in the model for the interference. The results are found in Figure 3 from which we conclude that the interference experienced by a user in the network is approximately normal distributed. This approximation is better for higher channel utilizations, which is a term describing the fraction of the available 

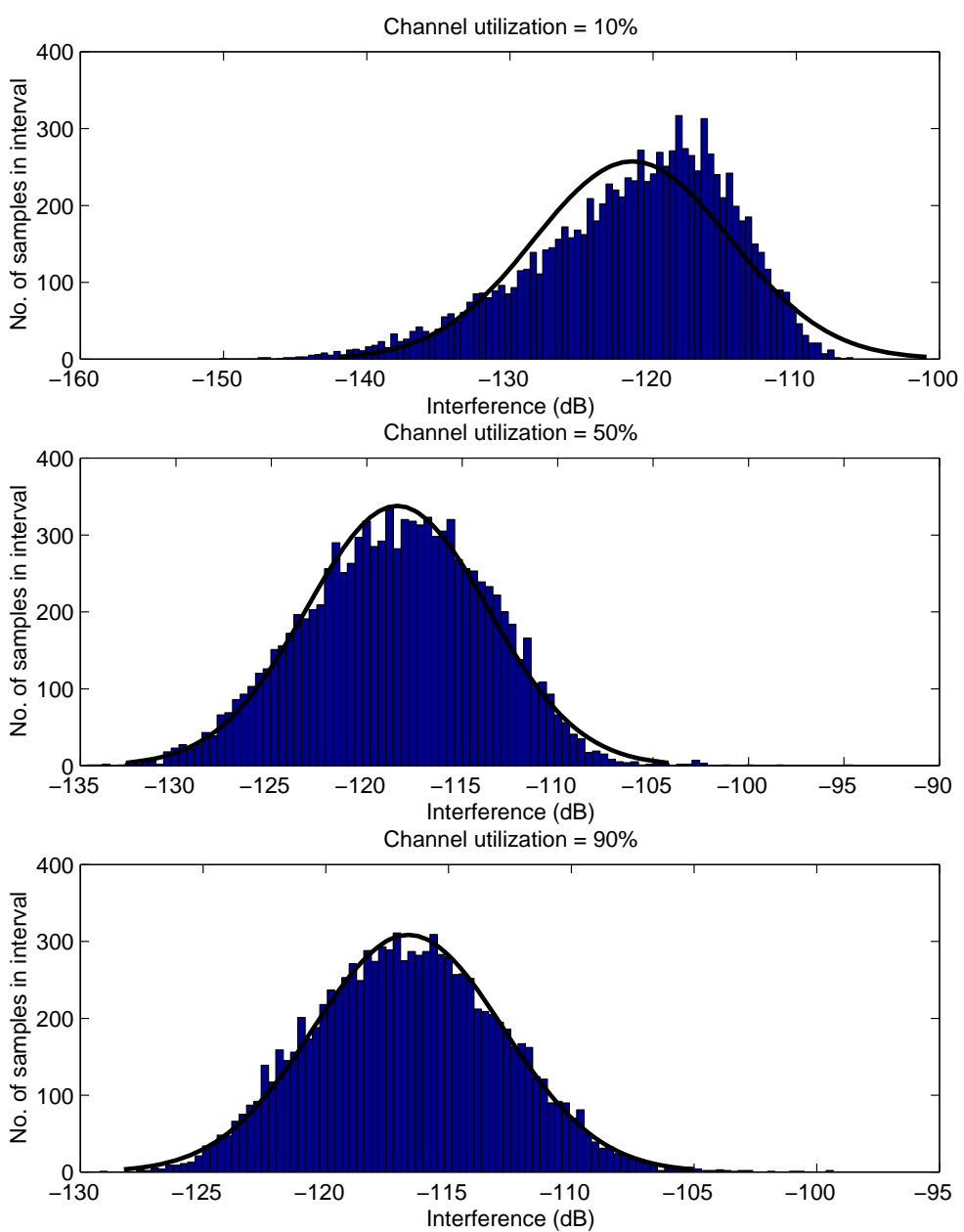

Figure 3: Interference distribution in a random frequency hopping network. The channel utilization (or interference load) refers to the extent to which the channel is used.

communication links which are occupied. This result proved to be relatively independent not only of network specific parameters such as cell radius and reuse, but also of the distribution of the transmitted powers.

The conclusion from this is that the interference distribution is characterized by its mean value $m_{I}$ and its standard deviation $\sigma_{I}$. This is supported by theoretical results, see [13, 12]. Our results hold on the burst time scale, i.e. for each burst the interference can be viewed as given by a realization of this normal distribution. Estimators used in previous work are only characterizing the interference by its mean value, see e.g. [1], and therefore this approach describes the interference more thoroughly.

Since the power can only be updated once every $0.48 \mathrm{~s}$, we can not control the power to compensate for the fast multi-path fading, which is relatively constant 
during a burst, but varies considerably over a frame. Instead, the time and interferer diversity will limit the influence of the fading.

The correlation distance for the shadow fading typically has the value $100 \mathrm{~m}$. For a vehicle traveling at $30 \mathrm{~m} / \mathrm{s}$, this distance will take $\approx 3.3 \mathrm{~s}$ to travel. This time is relatively long compared to the power update interval, $0.48 \mathrm{~s}$, and thus we expect to be able to compensate for these variations. The same conclusion holds for the path loss, which is slowly varying.

\subsection{Choice of Estimator}

According to the discussion in Section II.2.1 we want the estimator to estimate carrier power, $C$, together with the mean, $m_{I}$, and the standard deviation, $\sigma_{I}$, of the interference. These estimates will be denoted by $\hat{C}, \hat{m}_{I}$ and $\hat{\sigma}_{I}$. Together with the transmission power, these estimates can be used for calculating the path loss $\hat{g}=\hat{C}-p$ and the $\mathrm{C} / \mathrm{I}$ estimate $\left(\widehat{C / I}=\hat{C}-\hat{m}_{I}\right)$.

In GSM the measurement reports consist of RXLEV and RXQUAL. RXLEV is a signal strength measure, which has been quantized in 64 levels, and RXQUAL is a logarithmic measure of the bit error probability, quantized in 8 levels. As discussed in Section II.2.1, $x$ denotes the measurements and $\theta$ the parameters to be estimated

$$
\begin{aligned}
x & =(\mathrm{R} X L E V, \mathrm{R} X Q U A L)^{T} \\
\theta & =\left(C, m_{I}, \sigma_{I}\right)^{T} .
\end{aligned}
$$

The function $f(x, \theta)$ is found by simulations on the burst time scale using a model of the receiver together with the GSM standard.

Using simulations we can generate measurement reports as in the GSM system. In Figure 4 and 5, we find the results when estimating the carrier and C/I from the measurement reports with the estimator described above. Despite the loss of information in the quantization, the estimates are reasonably accurate.

\subsection{Choice of Quality Mapper}

It has been argued that FER is an appropriate objective measure of speech quality when considering a TDMA system [11]. Hence, we use this as the quality function, and using a model of the receiver we construct it similarly to [11]. A good estimate of FER can be obtained by repeating this process several times for every point in the grid and forming the average.

The result of the procedure above is shown in Figure 6. This shows how the FER depends on the different estimated parameters.

For the quality mapper algorithm, a specified maximum allowable FER is input by the operator. In this example we choose FER $=2 \%$, see [11]. Using the procedure outlined in Section II.2.2 for this quality specification, we obtain 
a.

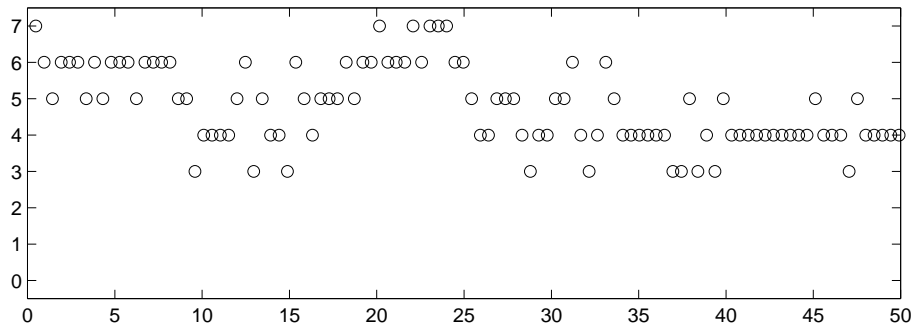

b.

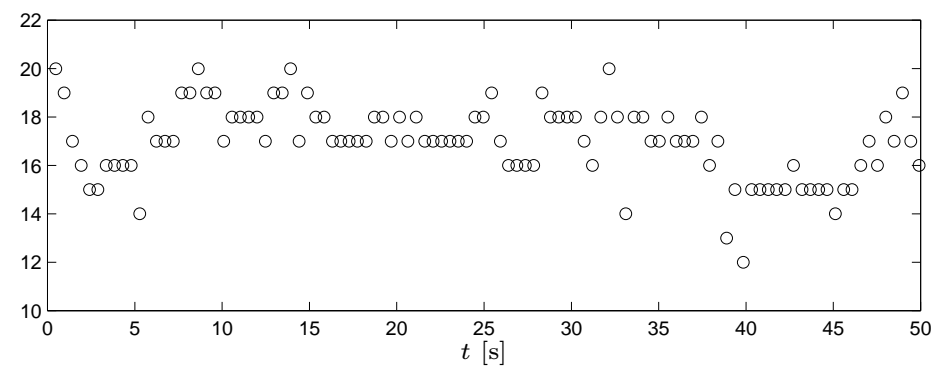

Figure 4: Measurement reports, consisting of a. RXQUAL and b. RXLEV, describing the perceived quality and signal strength respectively. These values are fed to the estimator.

a look-up table, see Figure 7. By controlling the carrier power such that we exceed the threshold in this figure, we can achieve the specified quality.

In a normal traffic situation, a C/I equal to $10 \mathrm{~dB}$ corresponds to an acceptable speech quality. For comparison purposes we can study such a normal traffic situation using simulations, from which it is found that the estimated standard deviation of the interference, $\sigma_{I}$, is typically $6 \mathrm{~dB}$, which is also indicated by the Monte Carlo simulations in Figure 3. As demonstrated by Figure 7, this estimated $\sigma_{I}$ yields a target $\mathrm{C} / \mathrm{I}$ of $10 \mathrm{~dB}$ in order to achieve $\mathrm{FER}=2 \%$, and thus our approach in this typical case covers the traditional opinion that a $\mathrm{C} / \mathrm{I}$ of $10 \mathrm{~dB}$ corresponds to acceptable quality. However, we can conclude that a lower standard deviation will allow $\mathrm{C} / \mathrm{I}$ to be less than $10 \mathrm{~dB}$ without losing quality. Hence a lower power can be used, and this will enable additional gains in capacity. Conversely, for $\sigma_{I}>6 \mathrm{~dB}$ our algorithm recognizes the need to increase the $\mathrm{C} / \mathrm{I}$ above $10 \mathrm{~dB}$ in order to maintain acceptable quality.

\subsection{Choice of Power Control Algorithm}

In the current setting, the choice of power control algorithm is not depending on the type of cellular system. Therefore, we refer to the general case discussed in Section II.2.3 
a.

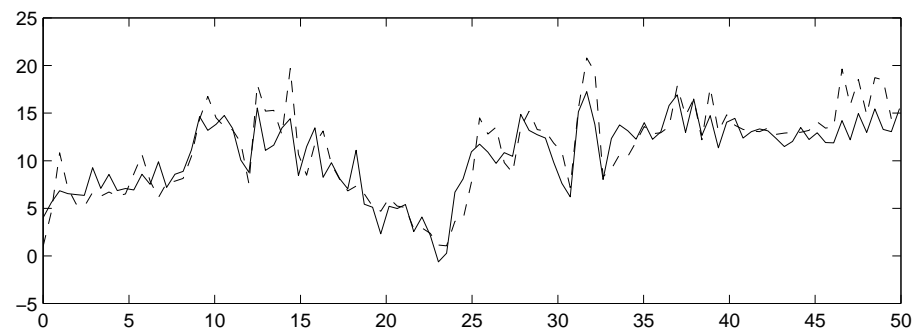

b.

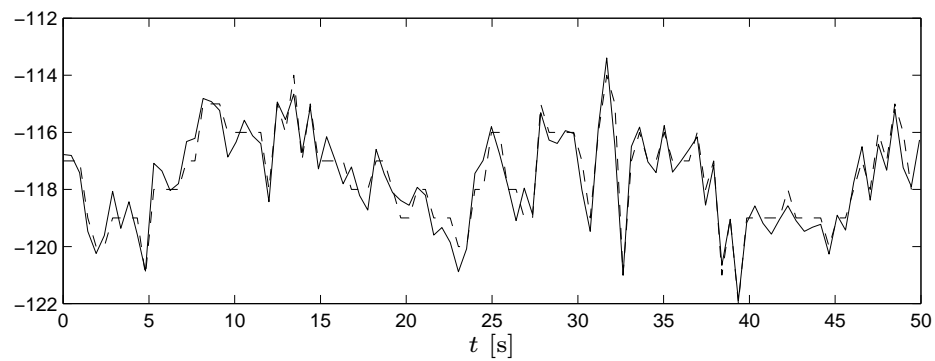

Figure 5: Given the measurement reports in Figure 4 the estimator extracts e.g. a. C/I and b. Carrier. The estimated values are shown by the solid lines, and the true values by dashed.

\section{Simulations}

In order to test if the concept with quality mapping is beneficial, we performed frequency hopping GSM system simulations. The situations on the different channels are not independent, and leakage between the channel has to be considered. Hence, we have to consider all the channels used in the system. Furthermore, the channel reuse is an important part and has to be taken into account.

The duration of one burst is $0.577 \mathrm{~ms}$ in GSM. During this time, the multipath fading is assumed to be constant. Furthermore assume that no user has a Line-of-Sight connection to the base station. Therefore the multi-path fading can be modeled as Rayleigh fading. The fading is correlated both in frequency and space, which has to be considered when simulating a system based on several carrier frequencies. Control signaling such as measurement reports and power control commands are transmitted more seldom. In total, 104 bursts are simulated each control signaling sample time $T_{c}$, resulting in $T_{c}=0.48 \mathrm{~s}$ (considering the fact that there are eight time slots). The simulation conditions are summarized in Table 1.

The percentage of satisfied users will be taken as performance measure. We consider a user to be satisfied if the corresponding average FER over time is below 0.02. In Figure 8, the number of satisfied users for different loads and algorithms can be found. These are shown relative to the number of satisfied users when all transmitters use the same constant power. The corresponding mean 


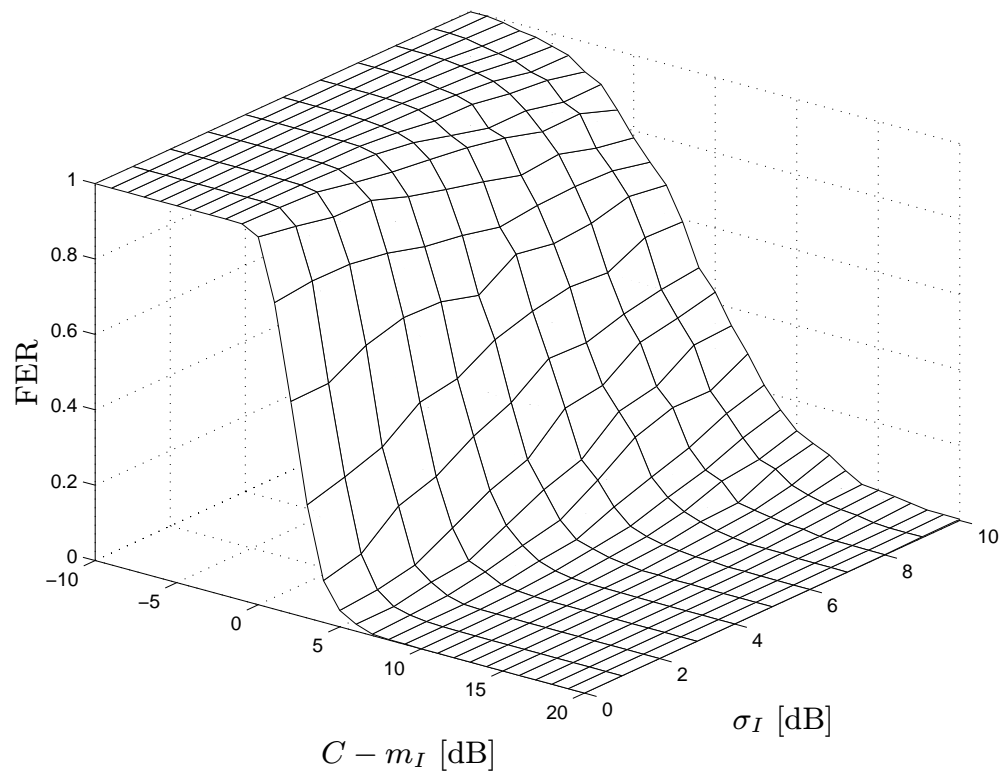

Figure 6: Frame erasure rate as a function of the carrier and the interference parameters for GSM.

powers used in the network are shown in Figure 9. By comparing the results for the I-controller with and without quality mapping, we find that additional capacity can be gained by including the quality mapper in the system.

\section{Summary}

We have presented a new concept of power control in cellular systems. Our power regulator is built up by three parts: The estimator, the quality mapper and the power control algorithm. We believe that the main features of this concept are that:

- It is ready for implementation in a second generation wireless system. The only component in the cellular system that has to be updated is the software in the base stations, where the output powers are computed. However, the concept is general and will be useful in a third generation wireless system as well.

- The transmission quality requirements can be specified using a measure that better reflects the actual quality perceived by the users. When considering frequency hopping GSM, the Frame Erasure Rate (FER) is an adequate quality measure.

- Using an estimator we are able to extract as much relevant information as possible from the measurement reports, such as the interference characteristics, the path loss, the carrier and the $\mathrm{C} / \mathrm{I}$. 


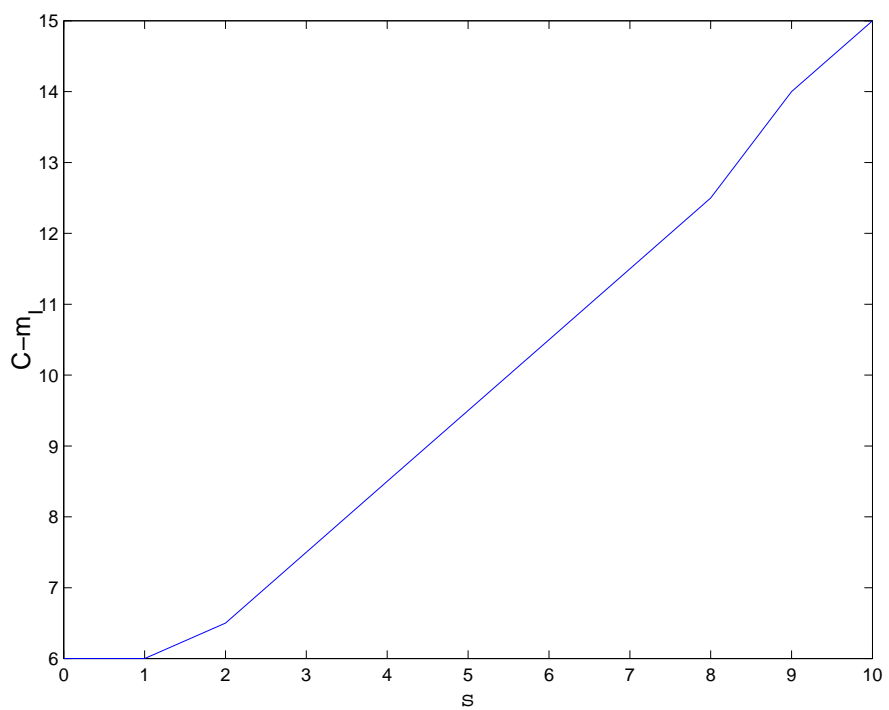

Figure 7: Threshold values for $\mathrm{C} / \mathrm{I}\left(C-m_{I}\right)$, based on the specification FER $=0.02$.

- When using the estimator and the quality mapper as described, the function of the power control algorithm is reduced to assigning transmitter power levels so that the estimated C/I will track the computed target $\mathrm{C} / \mathrm{I}$, in order to achieve the specified quality. Several of the power control algorithms suggested in the literature fit into this framework.

From the GSM example we find that using a quality mapper a lower power can be used when the standard deviation of the interference is low. On the contrary, when the standard deviation is high, this will be detected and the power will be increased in order to avoid speech quality degradations. System simulations show that additional capacity can be gained by using a quality mapper. 


\begin{tabular}{ll}
\hline Frequency band & $900 \mathrm{MHz}$ \\
No. of carrier freq. & 27 \\
Frequency reuse & $\mathrm{K}=9$ \\
Antennas & Sectorized \\
Cell radius & $1000 \mathrm{~m}$ \\
Cell layout & $5 \times 5$ clusters of 9 cells, \\
& employing wrap around \\
Adj. channel atten. & $-20 \mathrm{~dB}$ \\
Frequency hopping & Pseudo-random \\
Path-loss exponent & $\alpha=3.5$ \\
Shadow fading std.dev. & $\sigma_{s}=6 \mathrm{~dB}$ \\
Shadow fading corr.dist. & $d=100 \mathrm{~m}$ \\
Rayl. fading, avg. gain & $0 \mathrm{~dB}$ \\
Control sample interval & $T_{c}=0.48 \mathrm{~s}$ \\
Burst time & $0.577 \mathrm{~ms}$ \\
Mean MS speed & $50 \mathrm{~km} / \mathrm{h}$ \\
Downlink power & $13 \mathrm{dBW}$ (constant) \\
Uplink MS: & GSM class $4:$ \\
Uplink maximum power & $p_{\max }=3 \mathrm{dBW}$ \\
Uplink minimum power & $p_{\min }=-17 \mathrm{dBW}$ \\
Uplink quantization & $2 \mathrm{~dB}$ \\
\hline
\end{tabular}

Table 1: System simulation parameters.

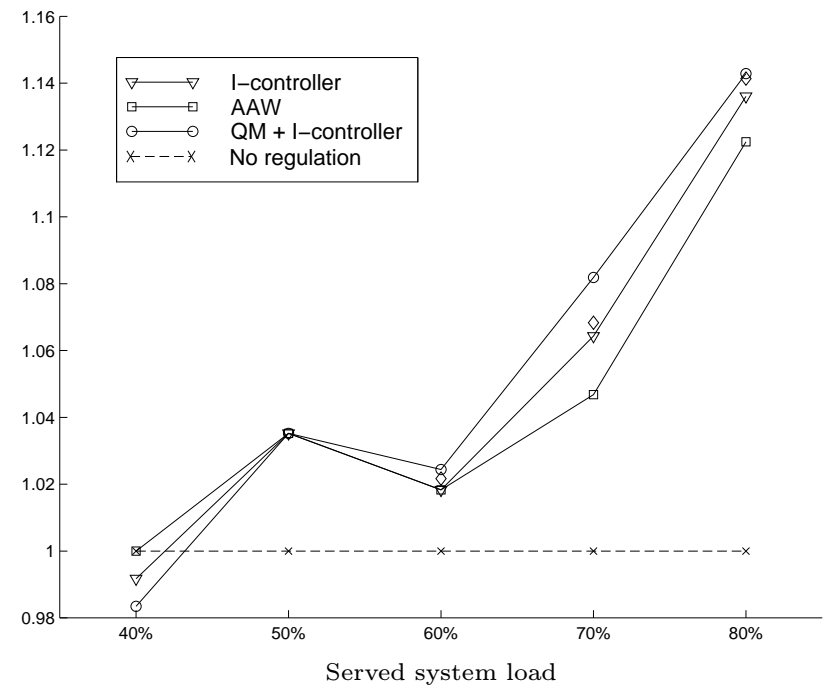

Figure 8: The number of satisfied customers for various served system load and algorithms relative to the number of satisfied customers when using constant maximum power. 


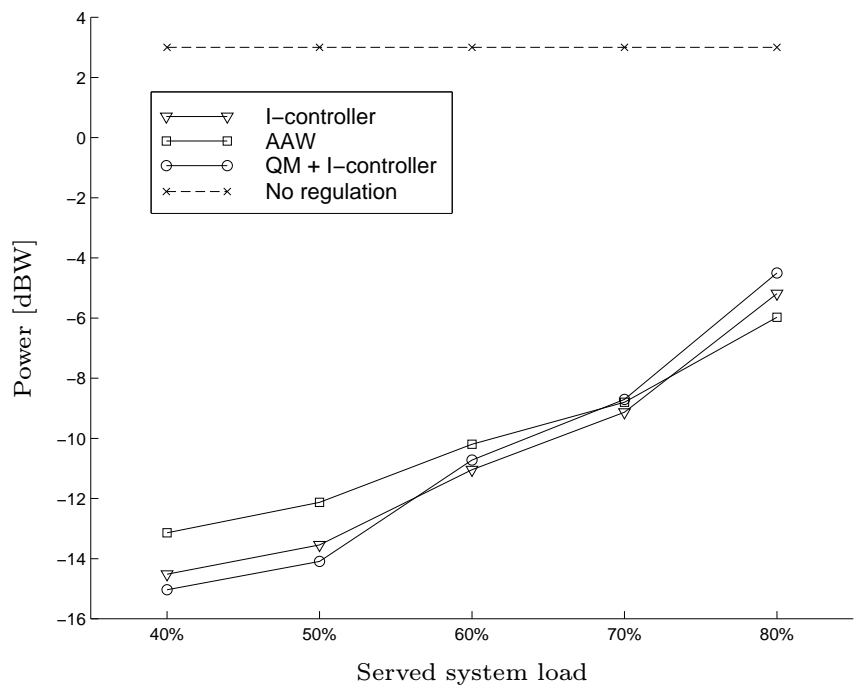

Figure 9: The mean power used in the network for various served system load and algorithms. 


\section{References}

[1] M. Almgren, H. Andersson, and K. Wallstedt. Power control in a cellular system. In Proc. IEEE Vehicular Technology Conference, Stockholm, Sweden, June 1994.

[2] M. Andersin. Real-time estimation of the signal to interference ratio in cellular radio systems. In Proc. IEEE Vehicular Technology Conference, Phoenix, AZ, USA, May 1997.

[3] M. Andersin, Z. Rosberg, and J. Zander. Distributed discrete power control in cellular PCS. Wireless Personal Communications, 6(3), 1998.

[4] J. Blom and F. Gunnarsson. Power Control in Cellular Radio Systems. Licenciate Thesis, Linköpings universitet, Sweden, June 1998. Available at http://www. control.isy.liu.se.

[5] G. J. Foschini and Z. Miljanic. A simple distributed autonomus power control algorithm and its covergence. IEEE Transactions on Vehicular Technology, 42(4), 1993.

[6] S. A. Grandhi, R. Vijayan, and D. J. Goodman. Distributed power control in cellular radio systems. IEEE Transactions on Communications, 42(2), 1994.

[7] S. A. Grandhi, J. Zander, and R. Yates. Constrained power control. Wireless Personal Communications, 2(1), 1995.

[8] F. Gunnarsson, J. Blom, and F. Gustafsson. Method and system for quality-based power control in cellular communications systems. US Patent Application No. 09/031,635, 1998.

[9] F. Gunnarsson, J. Blom, and F. Gustafsson. Power control in cellular systems subject to constraints and time delays. In Proc. IEEE Global Telecommunications Conference, Sydney, Australia, November 1998.

[10] E.L. Lehmann. Theory of Point Estimation. Statistical/Probability series. Wadsworth \& Brooks/Cole, 1991.

[11] H. Olofsson. Improved quality estimation for use in simulation of wireless TDMA systems. In Proc. IEEE International Conference on Universal Personal Communications, San Diego, CA, USA, October 1997.

[12] S. C. Schwartz and Y. S. Yeh. On the distribution function and moments of power sums with log-normal components. The Bell System Technical Journal, 61(7), 1982.

[13] G. L. Stüber. Principles of Mobile Communication. Kluwer Academic Publisher, Boston, MA, USA, 1996.

[14] M. Türkboylari and G.L. Stüber. An efficient algorithm for estimating the signal-to-interference ratio in TDMA cellular systems. IEEE Transactions on Communications, 46(6), 1998. 
[15] R. D. Yates. A framework for uplink power control in cellular radio systems. IEEE Journal on Selected Areas in Communications, 13(7), September 1995 .

[16] R. D. Yates, S. Gupta, C. Rose, and S. Sohn. Soft dropping power control. In Proc. IEEE Vehicular Technology Conference, Phoenix, AZ, USA, May 1997.

[17] J. Zander. Distributed cochannel interference control in cellular radio systems. IEEE Transactions on Vehicular Technology, 41(3), August 1992.

[18] J. Zander. Performance of optimum transmitter power control in cellular radio systems. IEEE Transactions on Vehicular Technology, 41(1), February 1992. 